$01.1 ; 04.4$

\title{
Подъем пылевых частиц при воздействии лазерного излучения на хондритовую мишень и возможность моделирования плазменно-пылевых процессов у поверхности Луны
}

\author{
(С) И.Н. Бурдонский ${ }^{1,2}$, А.Г. Леонов ${ }^{1}$, В.Н. Юфаa ${ }^{1,2}$, А.П. Голубь ${ }^{3}$, С.И. Попель ${ }^{3, \uparrow, ~ А . М . ~ С а д о в с к и и ̆ ~}{ }^{3}$ \\ ${ }^{1}$ Московский физико-технический институт (Национальный исследовательский университет), \\ Долгопрудный, Московская обл., Россия \\ ${ }^{2}$ АО „ГНЦ РФ Тринити“, Москва, Троицк, Россия \\ ${ }^{3}$ Институт космических исследований РАН, Москва, Россия \\ ฯ E-mail: popel@iki.rssi.ru
}

Поступило в Редакцию 28 апреля 2020 г.

В окончательной редакции 22 июля 2020 г.

Принято к публикации 23 июля 2020 г.

\begin{abstract}
Приведены результаты первого исследования по экспериментальному моделированию подъема пылевых частиц ударной волной над поверхностью мишени, выполненного на установке „Сатурн“. Исследовано взаимодействие лазерного излучения с пористой хондритовой мишенью, на поверхности которой содержатся мелкодисперсные частицы талька. Результаты экспериментального моделирования могут применяться для описания подъема частиц пыли из зон нелинейных и линейных упругих деформаций вещества реголита, характеризующих ударное воздействие метеороида на поверхность Луны.
\end{abstract}

Ключевые слова: экспериментальное моделирование, лазерное излучение, ударная волна, пылевые частицы, Луна.

DOI: $10.21883 /$ PJTF.2020.20.50157.18362

В настоящее время существенное внимание уделяется изучению пыли и пылевой плазмы над Луной [1-6]. В России готовятся миссии „Луна- $25,26,27^{\circ 6}$ и т.д. На посадочных модулях станций „Луна-25“ и „Луна-27“ предполагается разместить аппаратуру, которая будет исследовать свойства пыли и пылевой плазмы над поверхностью Луны. В миссии NASA LADEE [7], запущенной в 2013 г., пылевая среда изучалась в широком диапазоне высот над Луной посредством наблюдений с орбиты.

Важный источник пыли над поверхностью Луны удары метеороидов, которые приводят к подъему пылевых частиц (см., например, [4-6,8,9]). Учет ударов метеороидов особенно важен для адекватного описания отрыва пылевых частиц от поверхности Луны [4]. Сложность системы, возникающей при ударе метеороида, не позволяет учесть все существенные процессы в расчетах. Поэтому важным представляется экспериментальное моделирование ударных процессов метеороидов о поверхность Луны. В настоящей работе приводятся результаты диагностики перемещения пылевых частиц с поверхности мишени на установке „Сатурн“ [10]. Для моделирования ударов метеороидов о поверхность Луны используются импульсы сфокусированного излучения $\mathrm{Nd}^{3+}$-лазера.

Схема проведения экспериментов приведена на рис. 1. На лицевую поверхность хондритовой мишени наносится тонкий слой мелкодисперсного порошка талька, моделирующий пылевой слой на поверхности Луны. Размер частиц талька находится в пределах от 15 до $25 \mu \mathrm{m}$.
Греющий импульс излучения неодимового лазера с энергией от 1 до $10 \mathrm{~J}$ и длительностью $30 \mathrm{~ns}$ фокусируется линзой $L_{f}$ на поверхность мишени под углом, близким к нормали, в пятно диаметром около $300 \mu \mathrm{m}$. В результате взаимодействия мощного лазерного излучения с мишенью образуется ударная волна, которая распространяется в материале мишени как в глубину, так и вдоль ее лицевой поверхности. Эта ударная волна в свою очередь сообщает частицам талька импульс (в том числе и в вертикальном направлении), под действием которого частицы отрываются от поверхности мишени. Таким образом, над мишенью образуется пылевой слой. Излучение зондирующего лазера с энергией импульса $E \sim 20 \mu \mathrm{J}$, длиной волны $\lambda=0.532 \mu$ т и длительностью импульса $\tau=7 \mathrm{~ns}$ направляется сбоку вдоль лицевой поверхности мишени. Его размер в сечении $A-A$ составляет $3.5 \mathrm{~mm}$. Минимальное время задержки зондирующего импульса относительно греющего импульса составляет $1.3 \mu \mathrm{s}$. Изображение плоскости $A-A$ с помощью телескопа $L_{1}-L_{2}$ с увеличением формируется на CCD-матрице. Для точного определения коэффициента увеличения телескопа $(G=4.3)$ в плоскости $A-A$ установлена сетка с периодом $100 \mu \mathrm{m}$ и производится регистрация ее изображения на CCD-матрице в свете импульса зондирующего излучения. Кварцевая лампа ДРИ 1-100 предназначена для предварительной ионизации частиц талька в межэлектродном пространстве пластин $U$, на которые может быть подано постоянное или импульсное напряжение от 0.3 до $1 \mathrm{kV}$. Ее излучение через кварцевое окно вакуумной камеры посредством 


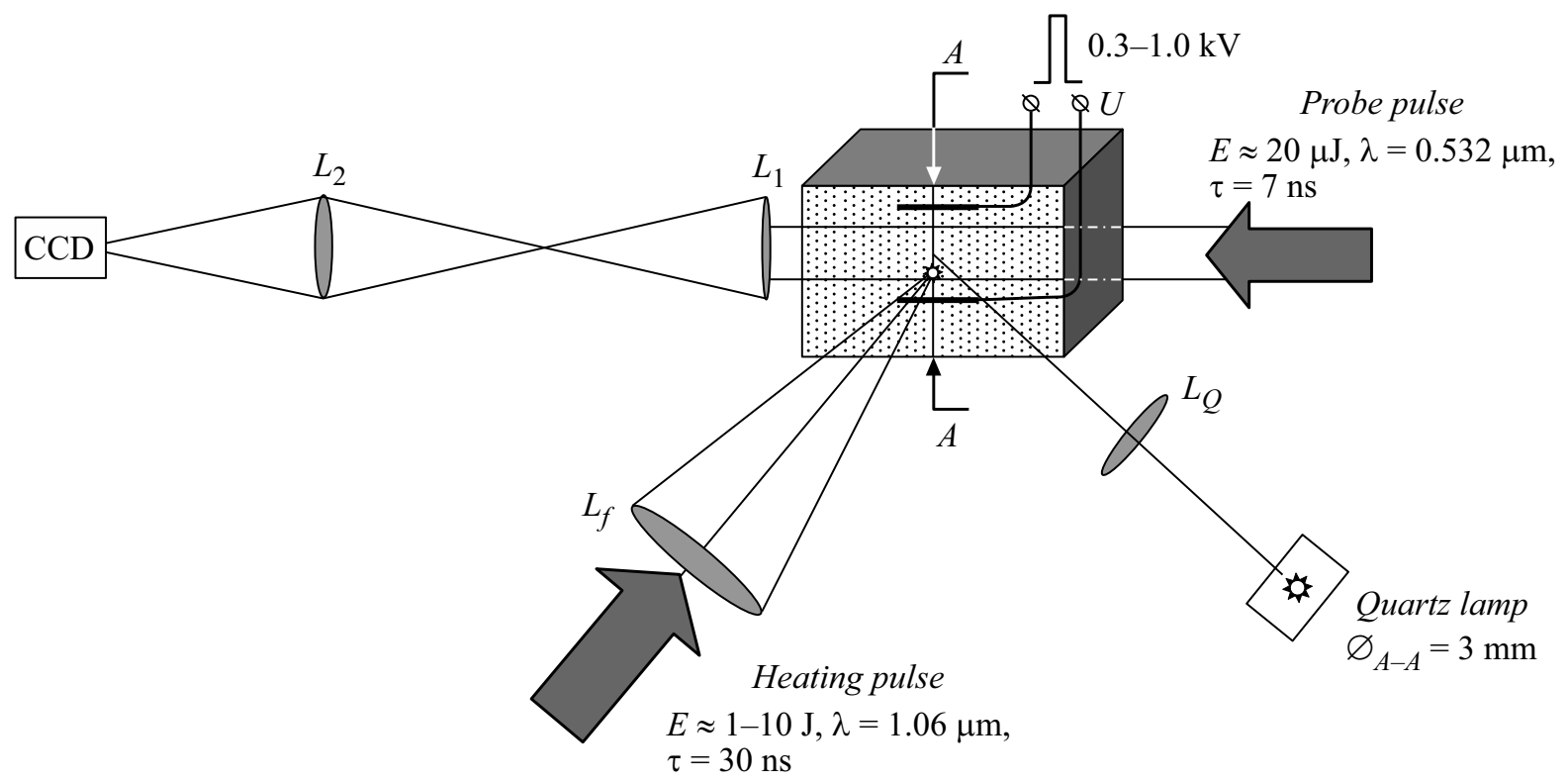

Рис. 1. Схема проведения экспериментов. $L_{f}$ - фокусирующая линза греющего импульса, Quartz lamp - кварцевая лампа ДРИ 1-100, $L_{Q}$ - кварцевая линза, $L_{1}, L_{2}$ - линзы телескопа, коэффициент увеличения телескопа $G \sim 5, A-A-$ изображение сетки с периодом $100 \mu \mathrm{m}$, полученное при импульсной подсветке зондирующим лазерным излучением, $U-$ клеммы подачи напряжения на металлические пластины, перпендикулярные фронтальной плоскости мишени.

кварцевой линзы $L_{Q}$ освещает с лицевой стороны мишени область диаметром около (или порядка) $4 \mathrm{~mm}$. Таким образом, заряженным пылевым частицам может сообщаться некоторая скорость дрейфа в направлении, перпендикулярном направлению зондирующего пучка.

При ударе метеороида о поверхность Луны вокруг эквивалентного центра метеороидного взрыва, расположенного на определенной глубине под поверхностью, формируются зоны (ср. с [9]), характеризующие происходящие при этом процессы: зона испарения вещества (I), зона плавления вещества (II), зона разрушения частиц, составляющих лунный реголит, и их необратимых деформаций (III), а также зона нелинейных упругих деформаций вещества реголита (IV), характеризуемая значениями давления в нелинейной звуковой волне, меньшими, чем динамический предел упругости. За зоной IV находится зона линейных упругих деформаций $(\mathrm{V})$, в которой звуковая волна может рассматриваться как линейная. Границы зон представляют собой части сфер, центр которых совпадает с эквивалентным центром метеороидного взрыва. Пусть скорость звука в невозмущенном реголите равна $c_{0}=300 \mathrm{~m} / \mathrm{s}$, ударник состоит из сплошного габброидного анортозита $[4,5,9,11]$, а веществом мишени является пористый габброидный анортозит. Тогда радиусы внешних границ зон I, II, III и IV под лунной поверхностью оказываются приблизительно равными [9] $r_{\mathrm{I}} \approx 0.31 u_{i}^{2 / 3} a_{i}$, $r_{\mathrm{II}} \approx 0.58 u_{i}^{2 / 3} a_{i}, \quad r_{\mathrm{III}} \approx 0.93 u_{i}^{2 / 3} a_{i}, \quad r_{\mathrm{IV}} \approx 1.3 u_{i}^{2 / 3} a_{i}$, где скорость $u_{i}$ метеороида вычисляется в $\mathrm{km} / \mathrm{s}$. Здесь $a_{i}$ радиус ударника.
При воздействии лазерного излучения большой интенсивности на поверхность твердого тела возникает абляция, заключающаяся в испарении и ионизации поверхностных слоев мишени, образовании плотной плазмы высокого давления и, как следствие, возбуждении гидродинамического движения (ударная волна) в неиспаренной части мишени [12-15]. Лазерное моделирование является вполне адекватным для описания ударного воздействия метеороида в области распространения ударной волны, причем лишь вне пятна облучения, в которое фокусируется греющий импульс излучения. Действительно, на больших расстояниях от точки метеороидного удара или от пятна лазерного воздействия ударная волна как бы забывает об источнике ее возникновения, и основным параметром, характеризующим источник возмущения, является энергия, идущая на образование ударной волны. Указанное обстоятельство позволяет воспользоваться законами подобия (аналогично возмущениям от точечного взрыва). При этом параметры, описывающие возмущения, оказываются связанными одними и теми же уравнениями, если нормировать все линейные размеры на корень кубический из полной выделившейся энергии вне зависимости от источника быстрого энерговыделения.

Таким образом, условие моделирования процессов, протекающих в зонах IV и V, образуемых в результате ударов метеороидов, имеет вид

$$
R \ll u_{i}^{2 / 3} a_{i}
$$

где $R$ - радиус пятна облучения, а $u_{i}$ выражается в km/s. Что касается зоны III, в которой происходит 


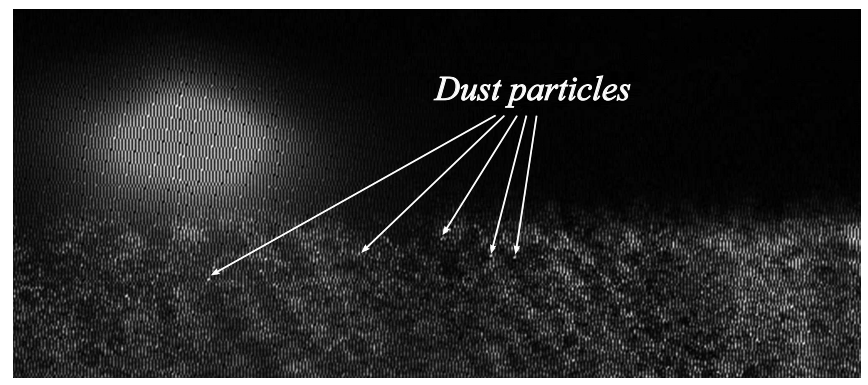

Рис. 2. Фронтальная поверхность мишени и плазменного факела в плоскости $A-A$ после прихода греющего лазерного импульса на мишень. Светящиеся точки - поднимающиеся в результате распространения ударной волны частицы талька.

разрушение частиц, составляющих лунный реголит, в ней лазерное моделирование уже дает не совсем верные результаты, поскольку значительная доля энергии лазерного импульса идет на нагрев среды, а при ударных процессах значительная часть энергии тратится на дробление материала мишени. Соотношение (1) оказывается справедливым для большинства метеороидов, падающих на поверхность Луны и имеющих размеры, бо́льшие или порядка $100 \mu \mathrm{m}$, т. е. именно тех метеороидов, процессы с участием которых вносят существенный вклад в формирование плазменно-пылевой системы. Теоретическое рассмотрение процессов, происходящих у поверхности Луны, при соударении с ней крупного метеороида (с размером, бо́льшим $100 \mu \mathrm{m}$ ) приводится в работе [9].

На рис. 2 представлено изображение фронтальной поверхности мишени и плазменного факела в плоскости $A-A$ через $1.3 \mu$ s после прихода греющего лазерного импульса на мишень. Изображение получено при импульсной подсветке зондирующим лазерным излучением. Предложенная схема эксперимента обеспечивает визуализацию пылевых частиц. Измерение скоростей частиц талька указывает на их значительные величины (вплоть до $5.5 \mathrm{~km} / \mathrm{s}$ ). Поднимающаяся пыль не подвергается непосредственному воздействию импульса греющего лазерного излучения, иначе пыль испарялась бы. Поэтому подъем частиц талька обусловлен взаимодействием частиц с ударной волной.

Имеется противоречие с результатами расчетов $[4,5,9]$, характеризующих ударное воздействие метеороида на поверхность Луны, согласно которым на границе между зонами II и III скорость выброса вещества определяется соотношением $u_{m t}=\sqrt{2 E_{c m t}} \approx 1.5 \mathrm{~km} / \mathrm{s}$, тогда как на границе между зонами I и II скорость выброса вещества $u_{v}=\sqrt{2 E_{c v}} \approx 6 \mathrm{~km} / \mathrm{s}$. Здесь $E_{c m t} \approx 1.1 \mathrm{MJ} / \mathrm{kg}$ - удельная пороговая внутренняя энергия полного плавления сплошного габброидного анортозита, $E_{c v} \approx 18 \mathrm{MJ} / \mathrm{kg}$ - удельная пороговая внутренняя энергия полного испарения (в условиях быстрой адиабатической механической разгрузки). Частицы реголита или их обломки из зон III-V имеют характерные размеры порядка 10-100 $\mu \mathrm{m}$ (максимум распределения по размерам находится в области 50-70 $\mu \mathrm{m})$ и поднимаются со скоростями, меньшими $1.5 \mathrm{~km} / \mathrm{s}$. Таким образом, согласно $[4,5,9]$, скорости частиц из зон III-V не могут достигать величины $5.5 \mathrm{~km} / \mathrm{s}$. Данный факт, по-видимому, связан с тем, что в $[4,5,9]$ не учитывался следующий эффект, возникающий в эксперименте.

Известно, что в пористом теле при выходе на свободную поверхность достаточно сильная ударная волна выбрасывает с большой скоростью газ, причем скорость разлетающегося газа значительно превосходит скорость выбрасываемого ударно-сжатого материала [16]. Этот эффект связан с очень неравномерным нагревом по массе сжатого вещества. На поверхностях контакта частиц, составляющих пористый материал, под действием больших сдвиговых напряжений за время схлопывания пор происходит разогрев тонких поверхностных слоев частиц, значительно более сильный, чем нагрев, происходящий равномерно по всей массе (в соответствии с ударной адиабатой пористого вещества). Обладая большой плотностью тепловой энергии, тонкие поверхностные слои частиц переходят в газообразное состояние и расширяются с большой скоростью в свободное пространство. Такая высокоскоростная газовая струя может захватить некоторые пылевые частицы, расположенные на поверхности пористого тела, и придать им скорость, значительно превосходящую скорость большинства пылевых частиц, выбрасываемых ударной волной. Повидимому, описанный процесс имел место в лазерном эксперименте.

Таким образом, в работе приведены результаты первого исследования по экспериментальному моделированию подъема пылевых частиц ударной волной над поверхностью мишени. В рамках исследований было обнаружено, что скорость пылевых частиц, приводимых в движение ударной волной, может приобретать достаточно большие значения (вплоть до $5.5 \mathrm{~km} / \mathrm{s}$ ), для объяснения которых требуется привлечение обычно не учитываемых при теоретическом описании механизмов подъема частиц. Результаты экспериментального моделирования могут применяться для описания подъема частиц пыли из зон нелинейных и линейных упругих деформаций вещества реголита, характеризующих ударное воздействие метеороида на поверхность Луны.

\section{Конфликт интересов}

Авторы заявляют, что у них нет конфликта интересов.

\section{Список литературы}

[1] Stubbs T.J., Vondrak R.R., Farrell W.M. // Adv. Space Res. 2006. V. 37. P. 59-66.

[2] Sternovsky Z., Chamberlin P., Horányi M., Robertson S., Wang X. // J. Geophys. Res. 2008. V. 113. P. A10104 (1-4).

[3] Stubbs T.J., Glenar D.A., Farrell W.M., Vondrak R.R., Collier M.R., Halekas J.S., Delory G.T. // Planet. Space Sci. 2011. V. 59. P. 1659-1664. 
[4] Попель С.И., Голубь А.П., Лисин Е.А., Извекова Ю.Н., Атаманюк Б., Дольников Г.Г., Захаров А.В., Зеленый Л.М. // Письма в ЖЭТФ. 2016. Т. 103. В. 9. С. 641-646.

[5] Попель С.И., Голубь А.П., Зеленый Л.М., Хораньи М. // Письма в ЖЭТФ. 2017. Т. 105. В. 10. С. 594-599.

[6] Berezhnoy A.A., Velikodsky Y.I., Zubko E., Iten M., Lena R., Sposetti S., Tereshchenko A.A., Popel S.I., Feoktistova E.A., Golub' A.P. // Planet. Space Sci. 2019. V. 177. P. 104689 (1-15).

[7] Horányi M., Szalay J.R., Kempf S., Schmidt J., Grün E., Srama R., Sternovsky Z. // Nature. 2015. V. 522. P. 324-326.

[8] Nemtchinov I.V., Shuvalov V.V., Artemieva N.A., Kosarev I.B., Popel S.I. // Int. J. Impact Eng. 2002. V. 27. P. 521-534.

[9] Попель С.И., Голубь А.П., Захаров А.В., Зеленый Л.М., Бережнной А.А., Зубко Е.С., Итен М., Лена Р., Спозетти С., Великодский Ю.И., Терещенко А.А., Атаманюк Б. // Письма в ЖЭТФ. 2018. Т. 108. В. 6. С. 379-387.

[10] Бурдонский И.Н., Гольцов А.Ю., Леонов А.Г., Макаров К.Н., Тимофеев И.С., Юба В.Н. // Вопр. атомной науки и техники. Сер. Термоядерный синтез. 2013. Т. 36. B. 2. C. $8-18$.

[11] Мелош Г. Образование ударных кратеров. М.: Мир, 1994. $336 \mathrm{c}$.

[12] Анисимов С.И., Прохоров А.М., Фортов В.Е. // УФН. 1984. T. 142. № 3. C. 395-434.

[13] Гуськов К.С., Гуськов С.Ю. // Квантовая электроника. 2001. T. 31. № 4. C. 305-310.

[14] Гуськов С.Ю. // ЖЭТФ. 2003. Т. 124. В. 6. С. 1271-1280.

[15] Гуськов С.Ю., Касперчик А., Писарчик Т., Бороздюк С., Уллшмид Й., Кроуски Е., Машек К., Пфейфер М., Скала Й., Писарчик П. // ЖЭТФ. 2007. Т. 132. В. 4. С. $907-$ 917.

[16] Беляков Г.В., Родионов В.Н., Самосадный В.П. // Физика горения и взрыва. 1977. № 4. С. 614-619. 